\title{
Crown Height and its Relation to Arch Width, Arch Length and Arch Perimeter in Ideal Occlusion
}

\author{
Fazal Shahid ${ }^{1}$, Mohammad Khursheed Alam ${ }^{1 *}$, Mohd Fadhli Khamis ${ }^{2}$ and Saqib Ali $^{3}$ \\ ${ }^{1}$ Department of Orthodontic, Malaysia \\ ${ }^{2}$ Department of Forensic Dentistry, Malaysia
}

${ }^{3}$ Department of Biomedical Dental Sciences, Saudi Arabia

Received: 眥: November 28, 2018; Published: 跚: December 14, 2018

*Corresponding author: Mohammad Khursheed Alam, Department of Orthodontic, Sakaka, Saudi Arabia

\begin{abstract}
Introduction: To investigate crown height for sexual dimorphism and its relation to arch length, arch perimeter and arch width (Inter canine, Inter premolar, Intermolar) groups of maxilla and mandible.

Material and Methods: Cross sectional study was done the data were collected from 128 subjects (64 males and 64 females with mean age 19.4 \pm 1.9 SD). Arch perimeter, arch length, crown height, and width of arch of both maxilla and mandible were acquired by digital calliper (Mitutoyo, Japan). Total variables measured were 4,855. Sex differences in the crown height were explored. Analysis of variance (ANOVA) was applied for evaluating the differences if any between arch perimeter, arch length, and arch width groups.

Results: Noteworthy larger mean values in males than females for crown height $(p \leq 0.05)$ for maxillary as well mandibular arches. No substantial differences were observed in average vs. large, average vs. small, large vs. small groups of arch length, arch perimeter and arch width (Inter canine, Inter premolar, Intermolar) groups $(\mathrm{p} \leq 0.05)$ for both maxillary and mandibular arches.

Conclusions: Our results specified sexual disparities in the height of crown. Crown height has no substantial relation to different arch perimeter, arch length, and arch width groups of both maxilla and mandible. Hence it might be useful for orthodontic case investigation and treatment of various malocclusion groups.
\end{abstract}

\section{Introduction}

Variations in tooth size and tooth size ratio have been connected with diverse ethnic foundations and occlusion status [1]. Sexual dimorphism in crown dimension has a relation and is influenced by humanoid sex genes and hormones [2]. Hereditary and ecological factors have strong effects on the human teeth [3]. In orthodontic diagnosis and treatment planning, great importance has been attached to evaluating the tooth size and its discrepancies on the global population [4]. For the achievement of pleasant smile and proper interdigitation, the preadjusted fixed orthodontic brackets should be ideally positioned. Thus, the crown height is of extreme value in orthodontic bonding. The teeth crown height has significant relation to facial stature, both can be swayed by orthodontic treatment [5] Therefore, during orthodontic planning to design a smile the macro, mini and micro-esthetics should be deliberated afore [6-10]. The crown height of incisors has tremendous effect on the smile of a patient, and leads an imperative part in facial charm [11]. Linear measurements have been incorporated into various dental model analyses to help the clinician for designing the most appropriate treatment plan [12]. Some studies have investigated race and sex differences. Other authors have suggested no significant differences in the tooth size ratio with different malocclusion [13].

However, the crown height was investigated in relation to facial height [14], facial appearances [15] and smile. According to our knowledge till date no one investigated the crown height in relation to the arch length, width, perimeter in normal occlusion. Current study goal was to explore the crown height of both maxilla and mandible, correspondingly to reconnaissance the following objectives for the first time in Pakistani population.

a) To evaluate crown height in relation to arch perimeter groups. 
b) To evaluate crown height in relation to arch length groups.

c) To evaluate crown height in relation to arch width groups in

d) Inter canine width

e) Inter premolar width

f) Intermolar width

g) To appraise amount of sexual dimorphism

\section{Methods}

Cross sectional study was done, the dental and oral examinations were carried out by careful selection of subjects from Pakistani population. University Sains Malaysia ethical committee ethical approval, bearing letter \# (USM/JEPeM/140376), before commencement of the study participants gave informed consent. The design and conduction of this study was in accordance with STROBE guideline (Strengthening the Reporting of Observational studies in Epidemiology), being applied in current manuscript [1620].

\section{Sample Size Calculation}

Size of the sample was calculated at a power of $80 \%$, using estimated standard deviations $0.60 \mathrm{~mm}$ [21], biologically significant mean difference $0.3 \mathrm{~mm}$, with equal sizes of sample [22]. Size of sample calculated was 64 females and 64 males, total 128 participants with mean age.

The inclusion as well the exclusion criteria:

\section{Inclusion Criteria}

a) All patient's origin was Pakistan determined by interviews, having common paternities plus ancestors without multi-ethnic marriages.

b) Subjects were aged 18-24 years.

c) Maxillary and mandibular arches which are well-aligned, showing normal development and growth patterns.

d) None of the participants had undertaken orthodontic treatment, with all thorough erupted permanent teeth, (3rd) third molars as exception. e) Class I molar, canine relationship with incisors plus Ideal occlusion in harmony with British Standards Institute [23].

f) No spacing, crowding or cross bite.

g) Straight profile identified by examination of the profile view.

h) Teeth following the ideal line of occlusion

i) No anomalies in craniofacial region.

\section{Exclusion Criteria}

a) Any restorations or caries interproximal.

b) Any supernumerary or missing teeth.

c) Anomalous morphology or size of teeth.

d) Extruded and intruded teeth.

e) Tooth wear affecting tooth size measurements.

f) Destruction to casts.

Dental and oral examinations were done with cautious selection of participants. For diminishing error and bias cross-examination of participants was done with skilled orthodontist along with dentists who contributed all over sittings of screening. Use of alginate impression material (Zhermack Orthoprint alginate ISO 1563 ADA 18 Italy) for obtaining dental impressions of both upper plus lower arches of individual participant and was then dispensed with hard plaster, Type III quick stone of China (Dental Stone) following instructions by manufacturer. Total variables measured were 4,855.

\section{Measurement of Crown Height and Arch Dimensions}

Both arches (Maxillary and mandibular) dental models of each participant was carefully selected according to inclusion and exclusion criteria. Both arches (Maxillary and mandibular) arch length, arch width, crown height were recorded by digital calliper (DC) (Mitutoyo, Japan) [24].

\section{Crown Height Measurement}

Crown heights were recorded as the greatest distance on buccal/labial surface from the occlusal/incisal line to cervical line parallel to occlusal plane [25] (Figure 1).
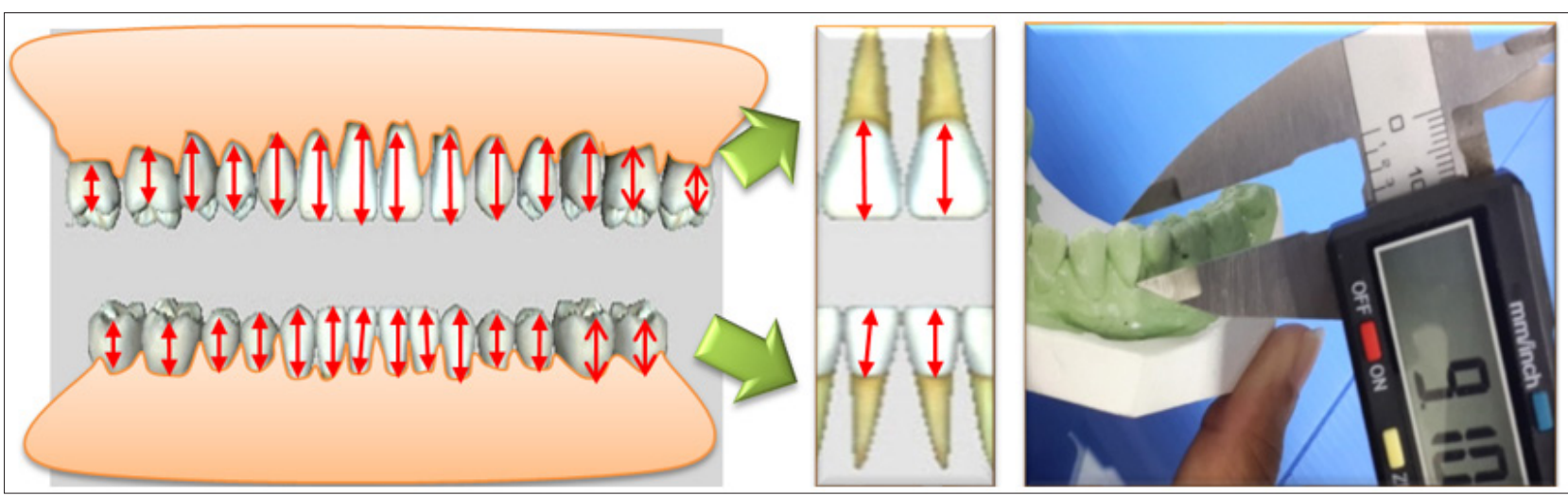

Figure 1: Crown height measurement via digital caliper. 


\section{Arch Dimensions Measurements}

Arch width of maxillary and mandibular variables (Figure 2a)

a) Maxillary and mandibular inter canine widths were obtained between tips of cusp.

b) Maxillary as well as mandibular interpremolar widths were obtained between cusp tips of first maxillary premolars [26].

c) Maxillary and mandibular intermolar widths were obtained between the mesiobuccally cusp tips the of first molars of maxillary and mandibular respectively [27].

\section{Arch Length, Arch Perimeter Measurements}

Arch Length: Arch length was obtained using triangular shaped lines amongst the mesiobuccally cusp tips of first permanent molars and central point between incisors of each respective arch (Figure 2b).

Arch Perimeter: Arch perimeter was measured as a segmental sum of linear lines on the left and right side of respective arches (Figure 2c).

Arch Length, Arch Perimeter and Arch Width Grouping: The participants were additionally grouped accordingly:

Figure 2: Arch dimension measurements.
a) Arch width
b) Arch length
c) Arch perimeter

a) Arch length groups (small, average and large).

The numbers of subjects for small, average and large arch length groups in the maxilla and mandible were 46,44 and 38 respectively.

b) Arch perimeter groups (small, average and large).

The number of subjects for small, average, and large arch perimeter groups in the maxilla and mandible were 44, 45 and 39 respectively.

c) Arch widths group (small, average and large)

The number of subjects for small, average, and large arch width groups in the maxilla and mandible were 44,45 and 39 respectively for (intercanine, interpremolar and intermolar width). These groupings were determined on data values of the Mean $\pm 2 \mathrm{SD}$, $>2$ SD and <2 SD grouped in the average, large and small group, correspondingly (Alam et al.).

\section{Error Study}

$20 \%$ of dental casts were randomly selected for interobserver errors. There was 2 weeks' time interval approximately between the first and second readings. The ME (method error) was analyzed by the Dalhberg's formula: $\mathrm{ME}=(\Sigma(\mathrm{x} 1-\mathrm{x} 2) 2 / 2(28)) 1 / 2 . \mathrm{x} 1$ being first and $\mathrm{x} 2$ being second measurement and $\mathrm{n}$ is the number of repeated measurements [9].

\section{Statistical Analyses}

Data verification and analysis was done statistically by use of IBM SPSS (Statistics Version 22.0, Armonk, NY: IBM Corp.) confidence level being set at $5 \%(P<0.05)$ for significance test. Application of independent $t$ tests for comparing mean values amongst males and females crown height in relation to all variables. Evaluation of values difference for arch width, length and perimeter was done with ANOVA (Analysis of variance).

\section{Results}

\section{Method Error}

Use of Dahlberg's formula for determining method error, not exceeding $0.05 \mathrm{~mm}$ for linear variables of teeth crown height. The collective errors for all the variables were small and was considered to be in acceptable limits [9].

\section{Sexual Disparities}

Tables $1 \& 2$ show the significant differences between the sexes with $p$ values ( $p \leq 0.001),(p \leq 0.01)$ and $(p \leq 0.05)$ in crown height for both arches (maxillary, mandibular) respectively. 
Table 1: Maxillary arch sexual disparities in crown height.

\begin{tabular}{|c|c|c|c|c|c|c|}
\hline \multirow[t]{2}{*}{ Variables } & \multirow[t]{2}{*}{ Sex } & \multirow[t]{2}{*}{ Mean } & \multirow[t]{2}{*}{ SD } & \multicolumn{2}{|c|}{$95 \%$ CI } & \multirow[t]{2}{*}{ P Value } \\
\hline & & & & Lower & Upper & \\
\hline \multirow[t]{2}{*}{11} & M & 9.44 & 0.67 & \multirow{2}{*}{.197} & \multirow{2}{*}{.659} & \multirow{2}{*}{.000} \\
\hline & $\mathrm{F}$ & 9.01 & 0.65 & & & \\
\hline \multirow[t]{2}{*}{12} & M & 7.98 & 0.71 & \multirow{2}{*}{.089} & \multirow{2}{*}{.541} & \multirow{2}{*}{.007} \\
\hline & $\mathrm{F}$ & 7.67 & 0.57 & & & \\
\hline \multirow[t]{2}{*}{13} & M & 8.94 & 0.86 & \multirow{2}{*}{.470} & \multirow{2}{*}{1.057} & \multirow{2}{*}{.000} \\
\hline & $\mathrm{F}$ & 8.18 & 0.82 & & & \\
\hline \multirow[t]{2}{*}{14} & M & 7.23 & 0.68 & \multirow{2}{*}{.413} & \multirow{2}{*}{.920} & \multirow{2}{*}{.000} \\
\hline & $\mathrm{F}$ & 6.57 & 0.77 & & & \\
\hline \multirow[t]{2}{*}{15} & M & 6.04 & 0.67 & \multirow{2}{*}{.179} & \multirow{2}{*}{.643} & \multirow{2}{*}{.001} \\
\hline & $\mathrm{F}$ & 5.62 & 0.65 & & & \\
\hline \multirow[t]{2}{*}{16} & M & 5.93 & 0.59 & \multirow{2}{*}{.186} & \multirow{2}{*}{.592} & \multirow{2}{*}{.000} \\
\hline & $\mathrm{F}$ & 5.54 & 0.57 & & & \\
\hline \multirow[t]{2}{*}{17} & M & 5.56 & 0.61 & & & \\
\hline & $\mathrm{F}$ & 5.08 & 0.55 & .214 & .079 & .000 \\
\hline 21 & M & 9.41 & 0.68 & 105 & 650 & مחم \\
\hline & $\mathrm{F}$ & 8.98 & 0.63 & .195 & .053 & .000 \\
\hline 22 & M & 7.93 & 0.72 & ס20 & 100 & 20? \\
\hline & $\mathrm{F}$ & 7.67 & 0.59 & (030 & .498 & .023 \\
\hline 23 & M & 8.89 & 0.87 & 357 & 930 & مחת \\
\hline & $\mathrm{F}$ & 8.25 & 0.76 & / 35 & .930 & .000 \\
\hline 24 & M & 7.26 & 0.73 & 316 & 060 & תمת \\
\hline & $\mathrm{F}$ & 6.65 & 0.77 & .40 & (00) & .000 \\
\hline 25 & M & 6.03 & 0.68 & 07 & {$[?$} & 10 \\
\hline & $\mathrm{F}$ & 5.72 & 0.62 & .079 & .534 & .009 \\
\hline 26 & M & 5.88 & 0.60 & 151 & 550 & 001 \\
\hline & $\mathrm{F}$ & 5.52 & 0.57 & .151 & .559 & .001 \\
\hline 27 & M & 5.51 & 0.62 & 178 & 568 & مח \\
\hline & F & 5.14 & 0.49 & .170 & (500. & .000 \\
\hline
\end{tabular}

Note: *sDI notation; F, female; M, male; CI, confidence interval; SD, standard deviation ( $\mathrm{p} \leq 0.001),(\mathrm{p} \leq 0.01)$ and $(\mathrm{p} \leq 0.05)$.

Table 2: Mandibular arch sexual disparities in crown height.

\begin{tabular}{|c|c|c|c|c|c|c|}
\hline \multirow[t]{2}{*}{ Variables } & \multirow[t]{2}{*}{ Sex } & \multirow[t]{2}{*}{ Mean } & \multirow[t]{2}{*}{ SD } & \multicolumn{2}{|c|}{$95 \%$ CI } & \multirow[t]{2}{*}{ P Value } \\
\hline & & & & Lower & Upper & \\
\hline \multirow[t]{2}{*}{31} & M & 7.50 & 0.61 & \multirow{2}{*}{.14} & \multirow{2}{*}{.59} & \multirow{2}{*}{.002} \\
\hline & $\mathrm{F}$ & 7.13 & 0.68 & & & \\
\hline \multirow[t]{2}{*}{32} & M & 7.48 & 1.00 & \multirow{2}{*}{.02} & \multirow{2}{*}{.61} & \multirow{2}{*}{.039} \\
\hline & $\mathrm{F}$ & 7.17 & 0.66 & & & \\
\hline \multirow[t]{2}{*}{33} & M & 8.87 & 0.87 & \multirow{2}{*}{.21} & \multirow{2}{*}{.79} & \multirow{2}{*}{.001} \\
\hline & $\mathrm{F}$ & 8.37 & 0.78 & & & \\
\hline \multirow[t]{2}{*}{34} & M & 7.48 & 0.70 & \multirow{2}{*}{.08} & \multirow{2}{*}{.53} & \multirow{2}{*}{.009} \\
\hline & $\mathrm{F}$ & 7.18 & 0.58 & & & \\
\hline \multirow[t]{2}{*}{35} & M & 6.49 & 0.50 & \multirow{2}{*}{.14} & \multirow{2}{*}{.50} & \multirow{2}{*}{.001} \\
\hline & $\mathrm{F}$ & 6.18 & 0.53 & & & \\
\hline \multirow[t]{2}{*}{36} & M & 6.28 & 0.48 & \multirow{2}{*}{.16} & \multirow{2}{*}{.48} & \multirow{2}{*}{.000} \\
\hline & $\mathrm{F}$ & 5.95 & 0.45 & & & \\
\hline
\end{tabular}




\begin{tabular}{|c|c|c|c|c|c|c|}
\hline 37 & M & 5.93 & 0.64 & \multirow{2}{*}{.16} & \multirow{2}{*}{.60} & \multirow{2}{*}{.001} \\
\hline & $\mathrm{F}$ & 5.55 & 0.60 & & & \\
\hline \multirow[t]{2}{*}{41} & $\mathrm{M}$ & 7.51 & 0.59 & \multirow{2}{*}{.15} & \multirow{2}{*}{.59} & \multirow{2}{*}{.001} \\
\hline & $\mathrm{F}$ & 7.15 & 0.66 & & & \\
\hline \multirow[t]{2}{*}{42} & $\mathrm{M}$ & 7.48 & 0.98 & \multirow{2}{*}{.00} & \multirow{2}{*}{.58} & \multirow{2}{*}{.051} \\
\hline & $\mathrm{F}$ & 7.19 & 0.65 & & & \\
\hline \multirow[t]{2}{*}{43} & $M$ & 8.91 & 0.87 & \multirow{2}{*}{.16} & \multirow{2}{*}{.76} & \multirow{2}{*}{.003} \\
\hline & $\mathrm{F}$ & 8.45 & 0.82 & & & \\
\hline \multirow[t]{2}{*}{44} & M & 7.57 & 0.70 & \multirow{2}{*}{-.03} & \multirow{2}{*}{.44} & \multirow{2}{*}{.088} \\
\hline & $\mathrm{F}$ & 7.37 & 0.63 & & & \\
\hline \multirow[t]{2}{*}{45} & $\mathrm{M}$ & 6.55 & 0.47 & \multirow{2}{*}{.10} & \multirow{2}{*}{.47} & \multirow{2}{*}{.003} \\
\hline & $\mathrm{F}$ & 6.26 & 0.57 & & & \\
\hline \multirow[t]{2}{*}{46} & $\mathrm{M}$ & 6.29 & 0.48 & \multirow{2}{*}{.09} & \multirow{2}{*}{.43} & \multirow{2}{*}{.004} \\
\hline & $\mathrm{F}$ & 6.03 & 0.50 & & & \\
\hline \multirow[t]{2}{*}{47} & M & 5.97 & 0.62 & \multirow{2}{*}{-2.34} & \multirow{2}{*}{1.22} & \multirow{2}{*}{.536} \\
\hline & $\mathrm{F}$ & 6.52 & 7.17 & & & \\
\hline
\end{tabular}

Note: *FDI notation; F, female; M, male; CI, confidence interval; SD, standard deviation ( $\mathrm{p} \leq 0.001),(\mathrm{p} \leq 0.01)$ and $(\mathrm{p} \leq 0.05)$.

Disparities in Relation to Arch Length, Perimeter and (intercanine, interpremolar and intermolar) groups in relation Widths Groups of Maxilla

Table 3-7 shows the maxillary arch perimeter, length, and width to crown height respectively. No noteworthy differences were observed in large vs. small, average vs. large, average vs. small arch groups $(\mathrm{p} \leq 0.05)$.

Table 3: Crown height in relation to upper arch perimeter groups.

\begin{tabular}{|c|c|c|c|c|c|c|}
\hline \multirow[t]{2}{*}{ Variables } & \multirow[t]{2}{*}{ UAPG } & \multirow[t]{2}{*}{ Mean } & \multirow[t]{2}{*}{ SD } & \multicolumn{2}{|c|}{$95 \% \mathrm{CI}$} & \multirow[t]{2}{*}{$\mathbf{P}$} \\
\hline & & & & Lower & Upper & \\
\hline \multirow[t]{3}{*}{11} & Av vs Sm & 9.23 & 0.61 & -0.34 & 0.37 & 1.000 \\
\hline & Av vs Lar & 9.21 & 0.75 & -0.39 & 0.36 & 1.000 \\
\hline & Lar vs Sm & 9.23 & 0.73 & -0.37 & 0.37 & 1.000 \\
\hline \multirow[t]{3}{*}{12} & Av vs Sm & 7.74 & 0.59 & -0.46 & 0.22 & 1.000 \\
\hline & Av vs Lar & 7.85 & 0.74 & -0.41 & 0.30 & 1.000 \\
\hline & Lar vs Sm & 7.91 & 0.66 & -0.18 & 0.52 & .724 \\
\hline \multirow[t]{3}{*}{13} & Av vs Sm & 8.54 & 0.67 & -0.38 & 0.56 & 1.000 \\
\hline & Av vs Lar & 8.46 & 0.98 & -0.74 & 0.25 & .693 \\
\hline & Lar vs Sm & 8.70 & 1.09 & -0.33 & 0.65 & 1.000 \\
\hline \multirow[t]{3}{*}{14} & Av vs Sm & 6.87 & 0.61 & -0.45 & 0.38 & 1.000 \\
\hline & Av vs Lar & 6.91 & 0.91 & -0.45 & 0.41 & 1.000 \\
\hline & Lar vs Sm & 6.92 & 0.87 & -0.38 & 0.48 & 1.000 \\
\hline \multirow[t]{3}{*}{15} & Av vs Sm & 5.74 & 0.53 & -0.52 & 0.19 & .795 \\
\hline & Av vs Lar & 5.90 & 0.86 & -0.34 & 0.41 & 1.000 \\
\hline & Lar vs Sm & 5.86 & 0.64 & -0.24 & 0.50 & 1.000 \\
\hline \multirow[t]{3}{*}{16} & Av vs Sm & 5.74 & 0.53 & -0.34 & 0.29 & 1.000 \\
\hline & Av vs Lar & 5.76 & 0.65 & -0.29 & 0.37 & 1.000 \\
\hline & Lar vs Sm & 5.71 & 0.67 & -0.35 & 0.30 & 1.000 \\
\hline \multirow[t]{3}{*}{17} & Av vs Sm & 5.27 & 0.51 & -0.43 & 0.21 & 1.000 \\
\hline & Av vs Lar & 5.38 & 0.71 & -0.28 & 0.39 & 1.000 \\
\hline & Lar vs Sm & 5.32 & 0.65 & -0.28 & 0.39 & 1.000 \\
\hline \multirow[t]{3}{*}{21} & Av vs Sm & 9.17 & 0.62 & -0.35 & 0.36 & 1.000 \\
\hline & Av vs Lar & 9.16 & 0.73 & -0.46 & 0.28 & 1.000 \\
\hline & Lar vs Sm & 9.25 & 0.72 & -0.29 & 0.45 & 1.000 \\
\hline
\end{tabular}




\begin{tabular}{|c|c|c|c|c|c|c|}
\hline 22 & Av vs Sm & 7.70 & 0.58 & -0.49 & 0.20 & .898 \\
\hline & Av vs Lar & 7.85 & 0.77 & -0.37 & 0.35 & 1.000 \\
\hline & Lar vs Sm & 7.86 & 0.65 & -0.20 & 0.51 & .847 \\
\hline \multirow[t]{3}{*}{23} & Av vs Sm & 8.54 & 0.63 & -0.39 & 0.51 & 1.000 \\
\hline & Av vs Lar & 8.48 & 0.90 & -0.72 & 0.23 & .627 \\
\hline & Lar vs Sm & 8.72 & 1.09 & -0.28 & 0.65 & 1.000 \\
\hline \multirow[t]{3}{*}{24} & Av vs Sm & 7.01 & 0.63 & -0.35 & 0.48 & 1.000 \\
\hline & Av vs Lar & 6.94 & 0.91 & -0.42 & 0.46 & 1.000 \\
\hline & Lar vs Sm & 6.92 & 0.89 & -0.52 & 0.34 & 1.000 \\
\hline \multirow[t]{3}{*}{25} & Av vs Sm & 5.79 & 0.52 & -0.50 & 0.18 & .788 \\
\hline & Av vs Lar & 5.95 & 0.80 & -0.30 & 0.42 & 1.000 \\
\hline & Lar vs Sm & 5.89 & 0.65 & -0.26 & 0.45 & 1.000 \\
\hline \multirow[t]{3}{*}{26} & Av vs Sm & 5.67 & 0.51 & -0.36 & 0.27 & 1.000 \\
\hline & Av vs Lar & 5.72 & 0.68 & -0.33 & 0.33 & 1.000 \\
\hline & Lar vs Sm & 5.72 & 0.64 & -0.28 & 0.37 & 1.000 \\
\hline \multirow[t]{3}{*}{27} & Av vs Sm & 5.27 & 0.47 & -0.46 & 0.14 & .585 \\
\hline & Av vs Lar & 5.43 & 0.63 & -0.17 & 0.46 & .762 \\
\hline & Lar vs Sm & 5.28 & 0.65 & -0.30 & 0.32 & 1.000 \\
\hline
\end{tabular}

Note: *FDI, notation; UAPG, upper arch perimeter group; CI, confidence interval; SD, standard deviation; SM, small arch length; AV, average arch length; LA, large arch length; CI, confidence interval; SD, standard deviation Mean diff., mean differences. Av vs Sm*,

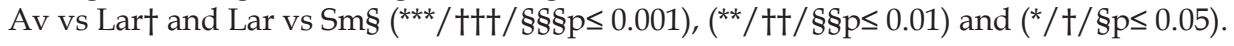

Table 4: Crown height in relation to upper arch length groups.

\begin{tabular}{|c|c|c|c|c|c|c|}
\hline \multirow{2}{*}{$\frac{\text { Variables* }}{\text { s }}$} & \multirow[t]{2}{*}{ UALG } & \multirow[t]{2}{*}{ Mean } & \multirow[t]{2}{*}{ SD } & \multicolumn{2}{|c|}{$95 \%$ CI } & \multirow[t]{2}{*}{$\mathbf{P}$} \\
\hline & & & & Lower & Upper & \\
\hline \multirow[t]{3}{*}{11} & Av vs Sm & 2.00 & 9.33 & 0.72 & -0.12 & 0.59 \\
\hline & Av vs Lar & 9.09 & 0.69 & -0.52 & 0.22 & .312 \\
\hline & Lar vs Sm & 9.24 & 0.66 & -0.46 & 0.28 & .970 \\
\hline \multirow[t]{3}{*}{12} & Av vs Sm & 7.86 & 0.71 & -0.21 & 0.47 & 1.000 \\
\hline & Av vs Lar & 7.73 & 0.64 & -0.52 & 0.19 & 1.000 \\
\hline & Lar vs Sm & 7.90 & 0.64 & -0.32 & 0.39 & .774 \\
\hline \multirow[t]{3}{*}{13} & Av vs Sm & 8.66 & 0.81 & -0.14 & 0.80 & 1.000 \\
\hline & Av vs Lar & 8.33 & 0.91 & -0.86 & 0.11 & .269 \\
\hline & Lar vs Sm & 8.71 & 1.01 & -0.44 & 0.53 & .184 \\
\hline \multirow[t]{3}{*}{14} & Av vs Sm & 6.94 & 0.72 & -0.23 & 0.59 & 1.000 \\
\hline & Av vs Lar & 6.76 & 0.83 & -0.69 & 0.15 & .841 \\
\hline & Lar vs Sm & 7.02 & 0.83 & -0.34 & 0.51 & .380 \\
\hline \multirow[t]{3}{*}{15} & Av vs Sm & 5.80 & 0.62 & -0.41 & 0.30 & 1.000 \\
\hline & Av vs Lar & 5.86 & 0.76 & -0.34 & 0.40 & 1.000 \\
\hline & Lar vs Sm & 5.82 & 0.70 & -0.35 & 0.39 & 1.000 \\
\hline \multirow[t]{3}{*}{16} & Av vs Sm & 5.79 & 0.53 & -0.20 & 0.43 & 1.000 \\
\hline & Av vs Lar & 5.68 & 0.68 & -0.40 & 0.26 & 1.000 \\
\hline & Lar vs Sm & 5.75 & 0.61 & -0.37 & 0.29 & 1.000 \\
\hline \multirow[t]{3}{*}{17} & Av vs Sm & 5.22 & 0.53 & -0.45 & 0.20 & 1.000 \\
\hline & Av vs Lar & 5.35 & 0.67 & -0.39 & 0.28 & 1.000 \\
\hline & Lar vs Sm & 5.40 & 0.66 & -0.15 & 0.51 & 1.000 \\
\hline \multirow[t]{3}{*}{21} & Av vs Sm & 9.29 & 0.72 & -0.11 & 0.59 & .569 \\
\hline & Av vs Lar & 9.05 & 0.68 & -0.55 & 0.18 & .299 \\
\hline & Lar vs Sm & 9.24 & 0.65 & -0.42 & 0.31 & .662 \\
\hline 22 & Av vs Sm & 7.87 & 0.69 & -0.12 & 0.56 & 1.000 \\
\hline
\end{tabular}

Cite this article: Fazal Shahid, Mohammad Khursheed A, Mohd Fadhli K, Saqib A. Crown Height and its Relation to Arch Width, Arch Length 


\begin{tabular}{|c|c|c|c|c|c|c|}
\hline & Av vs Lar & 7.66 & 0.65 & -0.58 & 0.12 & .375 \\
\hline & Lar vs Sm & 7.89 & 0.65 & -0.34 & 0.37 & .350 \\
\hline \multirow[t]{3}{*}{23} & Av vs Sm & 8.70 & 0.80 & -0.06 & 0.83 & 1.000 \\
\hline & Av vs Lar & 8.31 & 0.80 & -0.87 & 0.04 & .112 \\
\hline & Lar vs Sm & 8.73 & 0.99 & -0.43 & 0.49 & .087 \\
\hline \multirow[t]{3}{*}{24} & Av vs Sm & 7.04 & 0.80 & -0.17 & 0.65 & 1.000 \\
\hline & Av vs Lar & 6.80 & 0.80 & -0.66 & 0.19 & .488 \\
\hline & Lar vs Sm & 7.03 & 0.82 & -0.44 & 0.42 & .566 \\
\hline \multirow[t]{3}{*}{25} & Av vs Sm & 5.90 & 0.65 & -0.31 & 0.38 & 1.000 \\
\hline & Av vs Lar & 5.86 & 0.70 & -0.35 & 0.36 & 1.000 \\
\hline & Lar vs Sm & 5.86 & 0.66 & -0.40 & 0.32 & 1.000 \\
\hline \multirow[t]{3}{*}{26} & Av vs Sm & 5.71 & 0.55 & -0.30 & 0.33 & 1.000 \\
\hline & Av vs Lar & 5.69 & 0.66 & -0.34 & 0.31 & 1.000 \\
\hline & Lar vs Sm & 5.71 & 0.62 & -0.33 & 0.33 & 1.000 \\
\hline \multirow[t]{3}{*}{27} & Av vs Sm & 5.25 & 0.49 & -0.39 & 0.21 & 1.000 \\
\hline & Av vs Lar & 5.35 & 0.62 & -0.36 & 0.27 & 1.000 \\
\hline & Lar vs Sm & 5.39 & 0.65 & -0.17 & 0.45 & 1.000 \\
\hline
\end{tabular}

Note: *FDI, notation; UALG, upper arch length groups; SM, small arch length; AV, average arch length; LA, large arch length; CI,

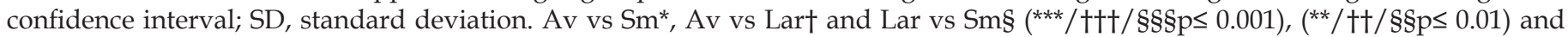
$(* / \dagger / \S p \leq 0.05)$.

Table 5: Crown height in relation to upper arch intercanine width groups.

\begin{tabular}{|c|c|c|c|c|c|c|}
\hline \multirow[t]{2}{*}{ Variables } & \multirow[t]{2}{*}{ UAICW } & \multirow[t]{2}{*}{ Mean } & \multirow[t]{2}{*}{ SD } & \multicolumn{2}{|c|}{$95 \%$ CI } & \multirow[t]{2}{*}{$\mathbf{P}$} \\
\hline & & & & Lower & Upper & \\
\hline \multirow[t]{3}{*}{11} & Av vs Sm & 9.26 & 0.63 & -0.30 & 0.43 & 1.000 \\
\hline & Av vs Lar & 9.19 & 0.75 & -0.39 & 0.35 & 1.000 \\
\hline & Lar vs Sm & 9.21 & 0.71 & -0.41 & 0.32 & 1.000 \\
\hline \multirow[t]{3}{*}{12} & Av vs $\mathrm{Sm}$ & 7.92 & 0.68 & -0.12 & 0.57 & .345 \\
\hline & Av vs Lar & 7.70 & 0.60 & -0.51 & 0.20 & .850 \\
\hline & Lar vs Sm & 7.85 & 0.69 & -0.41 & 0.28 & 1.000 \\
\hline \multirow[t]{3}{*}{13} & Av vs $\mathrm{Sm}$ & 8.66 & 0.75 & -0.24 & 0.72 & .654 \\
\hline & Av vs Lar & 8.42 & 1.07 & -0.67 & 0.32 & 1.000 \\
\hline & Lar vs Sm & 8.59 & 0.94 & -0.55 & 0.41 & 1.000 \\
\hline \multirow[t]{3}{*}{14} & Av vs Sm & 6.92 & 0.72 & -0.33 & 0.51 & 1.000 \\
\hline & Av vs Lar & 6.83 & 0.89 & -0.56 & 0.30 & 1.000 \\
\hline & Lar vs Sm & 6.95 & 0.79 & -0.38 & 0.45 & 1.000 \\
\hline \multirow[t]{3}{*}{15} & Av vs $\mathrm{Sm}$ & 5.80 & 0.66 & -0.36 & 0.36 & 1.000 \\
\hline & Av vs Lar & 5.81 & 0.81 & -0.45 & 0.30 & 1.000 \\
\hline & Lar vs Sm & 5.88 & 0.61 & -0.28 & 0.44 & 1.000 \\
\hline \multirow[t]{3}{*}{16} & Av vs Sm & 5.76 & 0.62 & -0.28 & 0.36 & 1.000 \\
\hline & Av vs Lar & 5.72 & 0.68 & -0.32 & 0.34 & 1.000 \\
\hline & Lar vs Sm & 5.72 & 0.53 & -0.37 & 0.27 & 1.000 \\
\hline \multirow[t]{3}{*}{17} & Av vs Sm & 5.39 & 0.54 & -0.20 & 0.46 & 1.000 \\
\hline & Av vs Lar & 5.27 & 0.79 & -0.36 & 0.31 & 1.000 \\
\hline & Lar vs Sm & 5.29 & 0.53 & -0.43 & 0.23 & 1.000 \\
\hline \multirow[t]{3}{*}{21} & Av vs Sm & 9.26 & 0.62 & -0.25 & 0.47 & 1.000 \\
\hline & Av vs Lar & 9.14 & 0.72 & -0.40 & 0.34 & 1.000 \\
\hline & Lar vs Sm & 9.17 & 0.73 & -0.44 & 0.28 & 1.000 \\
\hline \multirow[t]{2}{*}{22} & Av vs Sm & 7.85 & 0.71 & -0.24 & 0.46 & 1.000 \\
\hline & Av vs Lar & 7.74 & 0.59 & -0.43 & 0.29 & 1.000 \\
\hline
\end{tabular}




\begin{tabular}{|c|c|c|c|c|c|c|}
\hline & Lar vs Sm & 7.81 & 0.70 & -0.39 & 0.31 & 1.000 \\
\hline \multirow[t]{3}{*}{23} & Av vs Sm & 8.65 & 0.76 & -0.26 & 0.66 & .881 \\
\hline & Av vs Lar & 8.45 & 0.97 & -0.64 & 0.31 & 1.000 \\
\hline & Lar vs Sm & 8.61 & 0.90 & -0.49 & 0.42 & 1.000 \\
\hline \multirow[t]{3}{*}{24} & Av vs Sm & 7.01 & 0.82 & -0.25 & 0.59 & 1.000 \\
\hline & Av vs Lar & 6.84 & 0.88 & -0.61 & 0.26 & .991 \\
\hline & Lar vs Sm & 7.01 & 0.72 & -0.41 & 0.43 & 1.000 \\
\hline \multirow[t]{3}{*}{25} & Av vs Sm & 5.89 & 0.67 & -0.31 & 0.39 & 1.000 \\
\hline & Av vs Lar & 5.85 & 0.72 & -0.39 & 0.33 & 1.000 \\
\hline & Lar vs Sm & 5.89 & 0.62 & -0.35 & 0.35 & 1.000 \\
\hline \multirow[t]{3}{*}{26} & Av vs Sm & 5.75 & 0.62 & -0.23 & 0.40 & 1.000 \\
\hline & Av vs Lar & 5.67 & 0.69 & -0.33 & 0.32 & 1.000 \\
\hline & Lar vs Sm & 5.68 & 0.51 & -0.40 & 0.24 & 1.000 \\
\hline \multirow[t]{3}{*}{27} & Av vs Sm & 5.39 & 0.55 & -0.21 & 0.40 & 1.000 \\
\hline & Av vs Lar & 5.30 & 0.69 & -0.31 & 0.32 & 1.000 \\
\hline & Lar vs Sm & 5.29 & 0.51 & -0.41 & 0.21 & 1.000 \\
\hline
\end{tabular}

Note: *FDI, notation; UAICW, upper arch intercanine width groups; SM, small arch length; AV, average arch length; LA, large arch

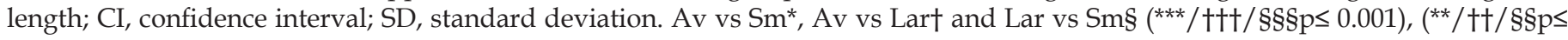
$0.01)$ and $(* / \dagger / \S p \leq 0.05)$.

Table 6: Crown height in relation to upper arch interpremolar width groups.

\begin{tabular}{|c|c|c|c|c|c|c|}
\hline \multirow{2}{*}{$\begin{array}{c}\text { Variables* } \\
\text { s }\end{array}$} & \multirow[t]{2}{*}{ UAPMW } & \multirow[t]{2}{*}{ Mean } & \multirow[t]{2}{*}{ SD } & \multicolumn{2}{|c|}{$95 \%$ CI } & \multirow[t]{2}{*}{$\mathbf{P}$} \\
\hline & & & & Lower & Upper & \\
\hline \multirow[t]{3}{*}{11} & Av vs $\mathrm{Sm}$ & 9.29 & 0.69 & -0.41 & 0.32 & 1.000 \\
\hline & Av vs Lar & 9.07 & 0.71 & -0.45 & 0.28 & 1.000 \\
\hline & Lar vs Sm & 9.29 & 0.67 & -0.24 & 0.49 & 1.000 \\
\hline \multirow[t]{3}{*}{12} & Av vs Sm & 7.90 & 0.71 & -0.23 & 0.47 & 1.000 \\
\hline & Av vs Lar & 7.67 & 0.68 & -0.44 & 0.26 & 1.000 \\
\hline & Lar vs Sm & 7.89 & 0.58 & -0.38 & 0.32 & 1.000 \\
\hline \multirow[t]{3}{*}{13} & Av vs Sm & 8.65 & 0.95 & -0.28 & 0.68 & .959 \\
\hline & Av vs Lar & 8.30 & 0.91 & -0.75 & 0.21 & .540 \\
\hline & Lar vs Sm & 8.70 & 0.87 & -0.41 & 0.55 & 1.000 \\
\hline \multirow[t]{3}{*}{14} & Av vs Sm & 6.93 & 0.83 & -0.42 & 0.42 & 1.000 \\
\hline & Av vs Lar & 6.78 & 0.84 & -0.51 & 0.34 & 1.000 \\
\hline & Lar vs Sm & 6.98 & 0.72 & -0.34 & 0.51 & 1.000 \\
\hline \multirow[t]{3}{*}{15} & Av vs Sm & 5.86 & 0.83 & -0.33 & 0.40 & 1.000 \\
\hline & Av vs Lar & 5.71 & 0.71 & -0.43 & 0.31 & 1.000 \\
\hline & Lar vs Sm & 5.91 & 0.49 & -0.34 & 0.39 & 1.000 \\
\hline \multirow[t]{3}{*}{16} & Av vs Sm & 5.81 & 0.61 & -0.23 & 0.40 & 1.000 \\
\hline & Av vs Lar & 5.60 & 0.69 & -0.52 & 0.13 & .429 \\
\hline & Lar vs Sm & 5.79 & 0.52 & -0.21 & 0.43 & 1.000 \\
\hline \multirow[t]{3}{*}{17} & Av vs Sm & 5.32 & 0.70 & -0.43 & 0.23 & 1.000 \\
\hline & Av vs Lar & 5.29 & 0.60 & -0.35 & 0.31 & 1.000 \\
\hline & Lar vs Sm & 5.35 & 0.58 & -0.22 & 0.44 & 1.000 \\
\hline \multirow[t]{3}{*}{21} & Av vs Sm & 9.28 & 0.67 & -0.39 & 0.33 & 1.000 \\
\hline & Av vs Lar & 9.04 & 0.71 & -0.49 & 0.24 & 1.000 \\
\hline & Lar vs Sm & 9.23 & 0.67 & -0.21 & 0.52 & .919 \\
\hline \multirow[t]{3}{*}{22} & Av vs Sm & 7.90 & 0.68 & -0.18 & 0.52 & .704 \\
\hline & Av vs Lar & 7.62 & 0.70 & -0.46 & 0.25 & 1.000 \\
\hline & Lar vs Sm & 7.85 & 0.61 & -0.42 & 0.28 & 1.000 \\
\hline
\end{tabular}




\begin{tabular}{|c|c|c|c|c|c|c|}
\hline 23 & Av vs Sm & 8.66 & 0.89 & -0.25 & 0.67 & .826 \\
\hline & Av vs Lar & 8.35 & 0.86 & -0.73 & 0.19 & .469 \\
\hline & Lar vs Sm & 8.68 & 0.86 & -0.40 & 0.53 & 1.000 \\
\hline \multirow[t]{3}{*}{24} & Av vs Sm & 7.02 & 0.88 & -0.29 & 0.56 & 1.000 \\
\hline & Av vs Lar & 6.80 & 0.85 & -0.51 & 0.34 & 1.000 \\
\hline & Lar vs Sm & 7.03 & 0.67 & -0.47 & 0.38 & 1.000 \\
\hline \multirow[t]{3}{*}{25} & Av vs Sm & 5.98 & 0.78 & -0.29 & 0.41 & 1.000 \\
\hline & Av vs Lar & 5.74 & 0.68 & -0.37 & 0.34 & 1.000 \\
\hline & Lar vs Sm & 5.89 & 0.49 & -0.40 & 0.31 & 1.000 \\
\hline \multirow[t]{3}{*}{26} & Av vs Sm & 5.73 & 0.63 & -0.30 & 0.34 & 1.000 \\
\hline & Av vs Lar & 5.64 & 0.67 & -0.45 & 0.19 & 1.000 \\
\hline & Lar vs Sm & 5.73 & 0.52 & -0.21 & 0.43 & 1.000 \\
\hline \multirow[t]{3}{*}{27} & Av vs Sm & 5.36 & 0.63 & -0.35 & 0.26 & 1.000 \\
\hline & Av vs Lar & 5.29 & 0.58 & -0.33 & 0.30 & 1.000 \\
\hline & Lar vs Sm & 5.33 & 0.55 & -0.25 & 0.37 & 1.000 \\
\hline
\end{tabular}

Note: *FDI, notation; UAPMW, upper arch interpremolar width groups; SM, small arch length; AV, average arch length; LA, large arch length; CI, confidence interval; SD, standard deviation. Av vs Sm*, Av vs Lar† and Lar vs Sm§ (***/†††/§§§p $\leq 0.001)$, $(* * / \dagger \dagger / \S \S p \leq 0.01)$ and $(* / \dagger / \S p \leq 0.05)$.

Table 7: Crown height in relation to upper arch intermolar width groups.

\begin{tabular}{|c|c|c|c|c|c|c|}
\hline \multirow{2}{*}{$\begin{array}{c}\text { Variables* } \\
\text { ss }\end{array}$} & \multirow[t]{2}{*}{ UAIMW } & \multirow[t]{2}{*}{ Mean } & \multirow[t]{2}{*}{ SD } & \multicolumn{2}{|c|}{$95 \%$ CI } & \multirow[t]{2}{*}{$\mathbf{P}$} \\
\hline & & & & Lower & Upper & \\
\hline \multirow[t]{3}{*}{11} & Av vs Sm & 9.17 & 0.68 & -0.41 & 0.32 & 1.000 \\
\hline & Av vs Lar & 9.21 & 0.71 & -0.45 & 0.28 & 1.000 \\
\hline & Lar vs Sm & 9.29 & 0.69 & -0.24 & 0.49 & 1.000 \\
\hline \multirow[t]{3}{*}{12} & Av vs $\mathrm{Sm}$ & 7.88 & 0.67 & -0.23 & 0.47 & 1.000 \\
\hline & Av vs Lar & 7.76 & 0.64 & -0.44 & 0.26 & 1.000 \\
\hline & Lar vs Sm & 7.85 & 0.68 & -0.38 & 0.32 & 1.000 \\
\hline \multirow[t]{3}{*}{13} & Av vs $\mathrm{Sm}$ & 8.60 & 0.83 & -0.28 & 0.68 & .959 \\
\hline & Av vs Lar & 8.41 & 0.99 & -0.75 & 0.21 & .540 \\
\hline & Lar vs Sm & 8.68 & 0.92 & -0.41 & 0.55 & 1.000 \\
\hline \multirow[t]{3}{*}{14} & Av vs Sm & 6.87 & 0.83 & -0.42 & 0.42 & 1.000 \\
\hline & Av vs Lar & 6.87 & 0.88 & -0.51 & 0.34 & 1.000 \\
\hline & Lar vs Sm & 6.96 & 0.69 & -0.34 & 0.51 & 1.000 \\
\hline \multirow[t]{3}{*}{15} & Av vs Sm & 5.83 & 0.71 & -0.33 & 0.40 & 1.000 \\
\hline & Av vs Lar & 5.80 & 0.78 & -0.43 & 0.31 & 1.000 \\
\hline & Lar vs Sm & 5.86 & 0.58 & -0.34 & 0.39 & 1.000 \\
\hline \multirow[t]{3}{*}{16} & Av vs Sm & 5.73 & 0.58 & -0.23 & 0.40 & 1.000 \\
\hline & Av vs Lar & 5.64 & 0.67 & -0.52 & 0.13 & .429 \\
\hline & Lar vs Sm & 5.84 & 0.57 & -0.21 & 0.43 & 1.000 \\
\hline \multirow[t]{3}{*}{17} & $\mathrm{Av}$ vs $\mathrm{Sm}$ & 5.25 & 0.64 & -0.43 & 0.23 & 1.000 \\
\hline & Av vs Lar & 5.35 & 0.64 & -0.35 & 0.31 & 1.000 \\
\hline & Lar vs Sm & 5.36 & 0.60 & -0.22 & 0.44 & 1.000 \\
\hline \multirow[t]{3}{*}{21} & Av vs Sm & 9.13 & 0.65 & -0.39 & 0.33 & 1.000 \\
\hline & Av vs Lar & 9.16 & 0.72 & -0.49 & 0.24 & 1.000 \\
\hline & Lar vs Sm & 9.29 & 0.69 & -0.21 & 0.52 & .919 \\
\hline \multirow[t]{3}{*}{22} & Av vs Sm & 7.88 & 0.66 & -0.18 & 0.52 & .704 \\
\hline & Av vs Lar & 7.71 & 0.64 & -0.46 & 0.25 & 1.000 \\
\hline & Lar vs Sm & 7.81 & 0.71 & -0.42 & 0.28 & 1.000 \\
\hline 23 & Av vs $\mathrm{Sm}$ & 8.62 & 0.75 & -0.25 & 0.67 & .826 \\
\hline
\end{tabular}




\begin{tabular}{|c|c|c|c|c|c|c|}
\hline & Av vs Lar & 8.41 & 0.95 & -0.73 & 0.19 & .469 \\
\hline & Lar vs Sm & 8.69 & 0.92 & -0.40 & 0.53 & 1.000 \\
\hline \multirow[t]{3}{*}{24} & Av vs Sm & 7.01 & 0.87 & -0.29 & 0.56 & 1.000 \\
\hline & Av vs Lar & 6.88 & 0.85 & -0.51 & 0.34 & 1.000 \\
\hline & Lar vs Sm & 6.97 & 0.68 & -0.47 & 0.38 & 1.000 \\
\hline \multirow[t]{3}{*}{25} & Av vs Sm & 5.91 & 0.71 & -0.29 & 0.41 & 1.000 \\
\hline & Av vs Lar & 5.85 & 0.72 & -0.37 & 0.34 & 1.000 \\
\hline & Lar vs Sm & 5.87 & 0.57 & -0.40 & 0.31 & 1.000 \\
\hline \multirow[t]{3}{*}{26} & Av vs Sm & 5.67 & 0.62 & -0.30 & 0.34 & 1.000 \\
\hline & Av vs Lar & 5.65 & 0.63 & -0.45 & 0.19 & 1.000 \\
\hline & Lar vs Sm & 5.78 & 0.58 & -0.21 & 0.43 & 1.000 \\
\hline \multirow[t]{3}{*}{27} & Av vs Sm & 5.29 & 0.56 & -0.35 & 0.26 & 1.000 \\
\hline & Av vs Lar & 5.34 & 0.61 & -0.33 & 0.30 & 1.000 \\
\hline & Lar vs Sm & 5.35 & 0.59 & -0.25 & 0.37 & 1.000 \\
\hline
\end{tabular}

Note: *FDI, notation; UAIMW, upper arch intermolar width groups; SM, small arch length; AV, average arch length; LA, large arch

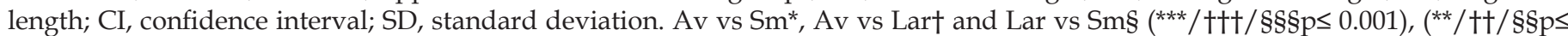
$0.01)$ and $(* / \dagger / \S p \leq 0.05)$.

Disparities in relation to arch length, perimeter and widths groups of mandibles

Table 8-12 show the mandibular arch perimeter, length, and

Table 8: Crown height in relation to lower arch perimeter groups.

\begin{tabular}{|c|c|c|c|c|c|c|}
\hline \multirow{2}{*}{$\begin{array}{c}\text { Variables* }^{*} \\
\text { ss }\end{array}$} & \multirow[t]{2}{*}{ LAPG } & \multirow[t]{2}{*}{ Mean } & \multirow[t]{2}{*}{ SD } & \multicolumn{2}{|c|}{$95 \%$ CI } & \multirow[t]{2}{*}{$\mathbf{P}$} \\
\hline & & & & Lower & Upper & \\
\hline \multirow[t]{3}{*}{31} & Av vs $\mathrm{Sm}$ & 7.34 & 0.68 & -0.29 & 0.41 & 1.000 \\
\hline & Av vs Lar & 7.28 & 0.70 & -0.39 & 0.31 & 1.000 \\
\hline & Lar vs Sm & 7.32 & 0.62 & -0.38 & 0.33 & 1.000 \\
\hline \multirow[t]{3}{*}{32} & Av vs Sm & 7.40 & 0.65 & -0.26 & 0.64 & .898 \\
\hline & Av vs Lar & 7.21 & 1.16 & -0.61 & 0.29 & 1.000 \\
\hline & Lar vs Sm & 7.37 & 0.66 & -0.49 & 0.42 & 1.000 \\
\hline \multirow[t]{3}{*}{33} & Av vs Sm & 8.60 & 0.77 & -0.51 & 0.39 & 1.000 \\
\hline & Av vs Lar & 8.66 & 0.92 & -0.39 & 0.52 & 1.000 \\
\hline & Lar vs Sm & 8.59 & 0.90 & -0.46 & 0.45 & 1.000 \\
\hline \multirow[t]{3}{*}{34} & Av vs Sm & 7.39 & 0.63 & -0.26 & 0.43 & 1.000 \\
\hline & Av vs Lar & 7.31 & 0.79 & -0.33 & 0.37 & 1.000 \\
\hline & Lar vs Sm & 7.29 & 0.55 & -0.46 & 0.24 & 1.000 \\
\hline \multirow[t]{3}{*}{35} & Av vs Sm & 6.30 & 0.56 & -0.27 & 0.29 & 1.000 \\
\hline & Av vs Lar & 6.29 & 0.60 & -0.40 & 0.16 & .891 \\
\hline & Lar vs Sm & 6.41 & 0.44 & -0.17 & 0.40 & 1.000 \\
\hline \multirow[t]{3}{*}{36} & Av vs Sm & 6.17 & 0.45 & -0.19 & 0.32 & 1.000 \\
\hline & Av vs Lar & 6.11 & 0.50 & -0.21 & 0.30 & 1.000 \\
\hline & Lar vs Sm & 6.06 & 0.53 & -0.37 & 0.15 & .912 \\
\hline \multirow[t]{3}{*}{37} & Av vs Sm & 5.77 & 0.60 & -0.39 & 0.28 & 1.000 \\
\hline & Av vs Lar & 5.83 & 0.69 & -0.12 & 0.56 & .349 \\
\hline & Lar vs Sm & 5.60 & 0.66 & -0.51 & 0.18 & .738 \\
\hline \multirow[t]{3}{*}{14} & Av vs Sm & 7.37 & 0.67 & -0.26 & 0.42 & 1.000 \\
\hline & Av vs Lar & 7.29 & 0.70 & -0.39 & 0.30 & 1.000 \\
\hline & Lar vs Sm & 7.33 & 0.60 & -0.38 & 0.31 & 1.000 \\
\hline
\end{tabular}

Cite this article: Fazal Shahid, Mohammad Khursheed A, Mohd Fadhli K, Saqib A. Crown Height and its Relation to Arch Width, Arch Length 


\begin{tabular}{|c|c|c|c|c|c|c|}
\hline 42 & Av vs $\mathrm{Sm}$ & 7.40 & 0.65 & -0.28 & 0.60 & 1.000 \\
\hline & Av vs Lar & 7.24 & 1.16 & -0.56 & 0.34 & 1.000 \\
\hline & Lar vs Sm & 7.35 & 0.59 & -0.50 & 0.40 & 1.000 \\
\hline \multirow[t]{3}{*}{43} & Av vs $\mathrm{Sm}$ & 8.69 & 0.83 & -0.47 & 0.45 & 1.000 \\
\hline & Av vs Lar & 8.69 & 0.92 & -0.45 & 0.48 & 1.000 \\
\hline & Lar vs Sm & 8.68 & 0.89 & -0.48 & 0.46 & 1.000 \\
\hline \multirow[t]{3}{*}{44} & Av vs Sm & 7.51 & 0.70 & -0.34 & 0.36 & 1.000 \\
\hline & Av vs Lar & 7.50 & 0.75 & -0.25 & 0.46 & 1.000 \\
\hline & Lar vs Sm & 7.39 & 0.54 & -0.48 & 0.23 & 1.000 \\
\hline \multirow[t]{3}{*}{45} & Av vs $\mathrm{Sm}$ & 6.34 & 0.56 & -0.38 & 0.18 & 1.000 \\
\hline & Av vs Lar & 6.44 & 0.60 & -0.29 & 0.28 & 1.000 \\
\hline & Lar vs Sm & 6.44 & 0.44 & -0.18 & 0.39 & 1.000 \\
\hline \multirow[t]{3}{*}{46} & Av vs Sm & 6.17 & 0.48 & -0.31 & 0.22 & 1.000 \\
\hline & Av vs Lar & 6.21 & 0.49 & -0.15 & 0.38 & .852 \\
\hline & Lar vs Sm & 6.09 & 0.55 & -0.34 & 0.20 & 1.000 \\
\hline \multirow[t]{3}{*}{47} & Av vs $\mathrm{Sm}$ & 7.11 & 8.71 & -1.44 & 3.84 & .820 \\
\hline & Av vs Lar & 5.92 & 0.66 & -2.45 & 2.90 & 1.000 \\
\hline & Lar vs Sm & 5.69 & 0.56 & -4.11 & 1.27 & .604 \\
\hline
\end{tabular}

Note: *FDI, notation; LAPG, lower arch perimeter groups; SM, small arch length; AV, average arch length; LA, large arch length; CI,

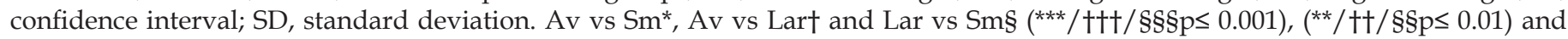
$(* / \dagger / \S p \leq 0.05)$.

Table 9: Crown height in relation to lower arch length groups.

\begin{tabular}{|c|c|c|c|c|c|c|}
\hline \multirow{2}{*}{$\begin{array}{c}\text { Variables* } \\
\mathrm{s}\end{array}$} & \multirow[t]{2}{*}{ LALG } & \multirow[t]{2}{*}{ Mean } & \multirow[t]{2}{*}{ SD } & \multicolumn{2}{|c|}{$95 \%$ CI } & \multirow[t]{2}{*}{ p } \\
\hline & & & & Lower & Upper & \\
\hline \multirow[t]{3}{*}{31} & Av vs Sm & 7.34 & 0.68 & -0.09 & 0.59 & .242 \\
\hline & Av vs Lar & 7.28 & 0.70 & -0.38 & 0.31 & 1.000 \\
\hline & Lar vs Sm & 7.32 & 0.62 & -0.56 & 0.14 & .427 \\
\hline \multirow[t]{3}{*}{32} & Av vs $\mathrm{Sm}$ & 7.40 & 0.65 & -0.40 & 0.50 & 1.000 \\
\hline & Av vs Lar & 7.21 & 1.16 & -0.45 & 0.47 & 1.000 \\
\hline & Lar vs Sm & 7.37 & 0.66 & -0.52 & 0.40 & 1.000 \\
\hline \multirow[t]{3}{*}{33} & $\mathrm{Av}$ vs $\mathrm{Sm}$ & 8.60 & 0.77 & -0.21 & 0.68 & .606 \\
\hline & Av vs Lar & 8.66 & 0.92 & -0.35 & 0.55 & 1.000 \\
\hline & Lar vs Sm & 8.59 & 0.90 & -0.78 & 0.12 & .233 \\
\hline \multirow[t]{3}{*}{34} & Av vs Sm & 7.39 & 0.63 & -0.37 & 0.33 & 1.000 \\
\hline & Av vs Lar & 7.31 & 0.79 & -0.37 & 0.33 & 1.000 \\
\hline & Lar vs Sm & 7.29 & 0.55 & -0.32 & 0.39 & 1.000 \\
\hline \multirow[t]{3}{*}{35} & Av vs Sm & 6.30 & 0.56 & -0.28 & 0.29 & 1.000 \\
\hline & Av vs Lar & 6.29 & 0.60 & -0.25 & 0.32 & 1.000 \\
\hline & Lar vs Sm & 6.41 & 0.44 & -0.33 & 0.25 & 1.000 \\
\hline \multirow[t]{3}{*}{36} & Av vs Sm & 6.17 & 0.45 & -0.23 & 0.29 & 1.000 \\
\hline & Av vs Lar & 6.11 & 0.50 & -0.21 & 0.31 & 1.000 \\
\hline & Lar vs Sm & 6.06 & 0.53 & -0.34 & 0.18 & 1.000 \\
\hline \multirow[t]{3}{*}{37} & Av vs Sm & 5.77 & 0.60 & -0.30 & 0.37 & 1.000 \\
\hline & Av vs Lar & 5.83 & 0.69 & -0.17 & 0.52 & .649 \\
\hline & Lar vs Sm & 5.60 & 0.66 & -0.55 & 0.13 & .413 \\
\hline \multirow[t]{3}{*}{14} & Av vs Sm & 7.37 & 0.67 & -0.10 & 0.58 & .269 \\
\hline & Av vs Lar & 7.29 & 0.70 & -0.36 & 0.32 & 1.000 \\
\hline & Lar vs Sm & 7.33 & 0.60 & -0.56 & 0.13 & .382 \\
\hline 42 & Av vs Sm & 7.40 & 0.65 & -0.39 & 0.50 & 1.000 \\
\hline
\end{tabular}




\begin{tabular}{|c|c|c|c|c|c|c|}
\hline & Av vs Lar & 7.24 & 1.16 & -0.39 & 0.51 & 1.000 \\
\hline & Lar vs Sm & 7.35 & 0.59 & -0.57 & 0.34 & 1.000 \\
\hline \multirow[t]{3}{*}{43} & Av vs $\mathrm{Sm}$ & 8.69 & 0.83 & -0.26 & 0.65 & .901 \\
\hline & Av vs Lar & 8.69 & 0.92 & -0.45 & 0.47 & 1.000 \\
\hline & Lar vs Sm & 8.68 & 0.89 & -0.67 & 0.26 & .870 \\
\hline \multirow[t]{3}{*}{44} & Av vs Sm & 7.51 & 0.70 & -0.34 & 0.37 & 1.000 \\
\hline & Av vs Lar & 7.50 & 0.75 & -0.39 & 0.33 & 1.000 \\
\hline & Lar vs Sm & 7.39 & 0.54 & -0.34 & 0.37 & 1.000 \\
\hline \multirow[t]{3}{*}{45} & Av vs Sm & 6.34 & 0.56 & -0.25 & 0.32 & 1.000 \\
\hline & Av vs Lar & 6.44 & 0.60 & -0.28 & 0.29 & 1.000 \\
\hline & Lar vs Sm & 6.44 & 0.44 & -0.33 & 0.25 & 1.000 \\
\hline \multirow[t]{3}{*}{46} & Av vs Sm & 6.17 & 0.48 & -0.27 & 0.25 & 1.000 \\
\hline & Av vs Lar & 6.21 & 0.49 & -0.15 & 0.38 & .842 \\
\hline & Lar vs Sm & 6.09 & 0.55 & -0.38 & 0.16 & .956 \\
\hline \multirow[t]{3}{*}{47} & Av vs Sm & 7.11 & 8.71 & -1.33 & 3.95 & .693 \\
\hline & Av vs Lar & 5.92 & 0.66 & -2.46 & 2.88 & 1.000 \\
\hline & Lar vs Sm & 5.69 & 0.56 & -4.21 & 1.16 & .514 \\
\hline
\end{tabular}

Note: *FDI, notation; LALG, lower arch length groups; SM, small arch length; AV, average arch length; LA, large arch length; CI,

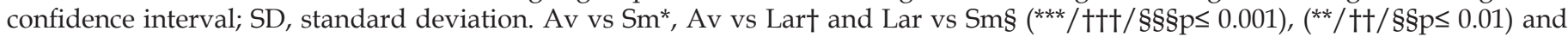
$(* / \dagger / \S p \leq 0.05)$.

Table 10: Crown height in relation to lower arch intercanine widths groups.

\begin{tabular}{|c|c|c|c|c|c|c|}
\hline \multirow{2}{*}{ Variables* } & \multirow{2}{*}{ LAICW } & \multirow{2}{*}{ Mean } & \multirow{2}{*}{ SD } & \multicolumn{2}{|c|}{$95 \% \mathrm{CI}$} & \multirow{2}{*}{$\mathbf{P}$} \\
\hline & & & & Lower & Upper & \\
\hline \multirow{3}{*}{31} & Av vs Sm & 7.38 & 0.65 & -0.09 & 0.59 & .242 \\
\hline & Av vs Lar & 7.17 & 0.76 & -0.38 & 0.31 & 1.000 \\
\hline & Lar vs Sm & 7.38 & 0.57 & -0.56 & 0.14 & .427 \\
\hline \multirow[t]{3}{*}{32} & Av vs Sm & 7.46 & 0.66 & -0.40 & 0.50 & 1.000 \\
\hline & Av vs Lar & 7.25 & 0.72 & -0.45 & 0.47 & 1.000 \\
\hline & Lar vs Sm & 7.26 & 1.12 & -0.52 & 0.40 & 1.000 \\
\hline \multirow[t]{3}{*}{33} & Av vs Sm & 8.73 & 0.88 & -0.21 & 0.68 & .606 \\
\hline & Av vs Lar & 8.49 & 0.82 & -0.35 & 0.55 & 1.000 \\
\hline & Lar vs Sm & 8.62 & 0.87 & -0.78 & 0.12 & .233 \\
\hline \multirow[t]{3}{*}{34} & Av vs $\mathrm{Sm}$ & 7.45 & 0.64 & -0.37 & 0.33 & 1.000 \\
\hline & Av vs Lar & 7.30 & 0.72 & -0.37 & 0.33 & 1.000 \\
\hline & Lar vs Sm & 7.22 & 0.61 & -0.32 & 0.39 & 1.000 \\
\hline \multirow[t]{3}{*}{35} & Av vs $\mathrm{Sm}$ & 6.32 & 0.57 & -0.28 & 0.29 & 1.000 \\
\hline & Av vs Lar & 6.32 & 0.59 & -0.25 & 0.32 & 1.000 \\
\hline & Lar vs Sm & 6.36 & 0.45 & -0.33 & 0.25 & 1.000 \\
\hline \multirow[t]{3}{*}{36} & Av vs Sm & 6.15 & 0.44 & -0.23 & 0.29 & 1.000 \\
\hline & Av vs Lar & 6.07 & 0.51 & -0.21 & 0.31 & 1.000 \\
\hline & Lar vs Sm & 6.12 & 0.53 & -0.34 & 0.18 & 1.000 \\
\hline \multirow[t]{3}{*}{37} & Av vs Sm & 5.76 & 0.61 & -0.30 & 0.37 & 1.000 \\
\hline & Av vs Lar & 5.72 & 0.68 & -0.17 & 0.52 & .649 \\
\hline & Lar vs Sm & 5.74 & 0.67 & -0.55 & 0.13 & .413 \\
\hline \multirow[t]{3}{*}{14} & Av vs Sm & 7.41 & 0.63 & -0.10 & 0.58 & .269 \\
\hline & Av vs Lar & 7.18 & 0.76 & -0.36 & 0.32 & 1.000 \\
\hline & Lar vs Sm & 7.39 & 0.55 & -0.56 & 0.13 & .382 \\
\hline 42 & Av vs Sm & 7.47 & 0.65 & -0.39 & 0.50 & 1.000 \\
\hline
\end{tabular}




\begin{tabular}{|c|c|c|c|c|c|c|}
\hline & Av vs Lar & 7.24 & 0.72 & -0.39 & 0.51 & 1.000 \\
\hline & Lar vs Sm & 7.27 & 1.10 & -0.57 & 0.34 & 1.000 \\
\hline \multirow[t]{3}{*}{43} & Av vs Sm & 8.78 & 0.86 & -0.26 & 0.65 & .901 \\
\hline & Av vs Lar & 8.54 & 0.86 & -0.45 & 0.47 & 1.000 \\
\hline & Lar vs Sm & 8.72 & 0.90 & -0.67 & 0.26 & .870 \\
\hline \multirow[t]{3}{*}{44} & Av vs Sm & 7.58 & 0.63 & -0.34 & 0.37 & 1.000 \\
\hline & Av vs Lar & 7.38 & 0.66 & -0.39 & 0.33 & 1.000 \\
\hline & Lar vs Sm & 7.44 & 0.72 & -0.34 & 0.37 & 1.000 \\
\hline \multirow[t]{3}{*}{45} & Av vs Sm & 6.42 & 0.54 & -0.25 & 0.32 & 1.000 \\
\hline & Av vs Lar & 6.40 & 0.61 & -0.28 & 0.29 & 1.000 \\
\hline & Lar vs Sm & 6.40 & 0.48 & -0.33 & 0.25 & 1.000 \\
\hline \multirow[t]{3}{*}{46} & Av vs Sm & 6.20 & 0.48 & -0.27 & 0.25 & 1.000 \\
\hline & Av vs Lar & 6.13 & 0.51 & -0.15 & 0.38 & .842 \\
\hline & Lar vs Sm & 6.15 & 0.53 & -0.38 & 0.16 & .956 \\
\hline \multirow[t]{3}{*}{47} & Av vs Sm & 5.83 & 0.58 & -1.33 & 3.95 & .693 \\
\hline & Av vs Lar & 5.79 & 0.64 & -2.46 & 2.88 & 1.000 \\
\hline & Lar vs Sm & 7.10 & 0.72 & -4.21 & 1.16 & .514 \\
\hline
\end{tabular}

Note: *FDI, notation; LALG, lower arch intercanine widths groups; SM, small arch length; AV, average arch length; LA, large arch

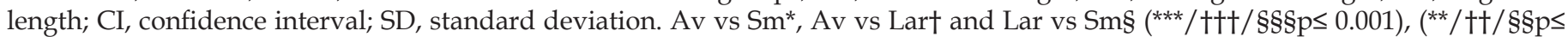
$0.01)$ and $(* / \dagger / \varsigma p \leq 0.05)$.

Table 11: Crown height in relation to lower arch interpremolar widths groups.

\begin{tabular}{|c|c|c|c|c|c|c|}
\hline \multirow{2}{*}{$\begin{array}{c}\text { Variables* } \\
\text { ss }\end{array}$} & \multirow{2}{*}{ LAPMW } & \multirow{2}{*}{ Mean } & \multirow[t]{2}{*}{ SD } & \multicolumn{2}{|c|}{$95 \% \mathrm{CI}$} & \multirow[t]{2}{*}{$\mathbf{P}$} \\
\hline & & & & Lower & Upper & \\
\hline \multirow[t]{3}{*}{31} & Av vs Sm & 7.44 & 0.63 & 0.01 & 0.69 & .043 \\
\hline & Av vs Lar & 7.09 & 0.72 & -0.65 & 0.03 & .081 \\
\hline & Lar vs Sm & 7.41 & 0.59 & -0.38 & 0.31 & 1.000 \\
\hline \multirow[t]{3}{*}{32} & Av vs Sm & 7.48 & 0.64 & -0.16 & 0.74 & .354 \\
\hline & Av vs Lar & 7.18 & 0.71 & -0.58 & 0.31 & 1.000 \\
\hline & Lar vs Sm & 7.32 & 1.14 & -0.61 & 0.29 & 1.000 \\
\hline \multirow[t]{3}{*}{33} & Av vs Sm & 8.73 & 0.84 & -0.24 & 0.66 & .796 \\
\hline & Av vs Lar & 8.52 & 0.85 & -0.32 & 0.58 & 1.000 \\
\hline & Lar vs Sm & 8.60 & 0.90 & -0.66 & 0.24 & .796 \\
\hline \multirow[t]{3}{*}{34} & Av vs Sm & 7.47 & 0.60 & -0.37 & 0.53 & 1.000 \\
\hline & Av vs Lar & 7.28 & 0.76 & -0.15 & 0.54 & .533 \\
\hline & Lar vs Sm & 7.24 & 0.59 & -0.30 & 0.38 & 1.000 \\
\hline \multirow[t]{3}{*}{35} & $\mathrm{Av}$ vs $\mathrm{Sm}$ & 6.36 & 0.53 & -0.58 & 0.11 & .311 \\
\hline & Av vs Lar & 6.29 & 0.60 & -0.21 & 0.36 & 1.000 \\
\hline & Lar vs Sm & 6.35 & 0.49 & -0.35 & 0.22 & 1.000 \\
\hline \multirow[t]{3}{*}{36} & Av vs Sm & 6.33 & 0.54 & -0.29 & 0.28 & 1.000 \\
\hline & Av vs Lar & 6.09 & 0.39 & -0.26 & 0.26 & 1.000 \\
\hline & Lar vs Sm & 6.08 & 0.51 & -0.34 & 0.17 & 1.000 \\
\hline \multirow[t]{3}{*}{37} & Av vs Sm & 5.74 & 0.59 & -0.34 & 0.35 & 1.000 \\
\hline & Av vs Lar & 5.73 & 0.68 & -0.34 & 0.34 & 1.000 \\
\hline & Lar vs Sm & 5.73 & 0.69 & -0.35 & 0.34 & 1.000 \\
\hline \multirow[t]{3}{*}{14} & Av vs Sm & 7.46 & 0.64 & 0.01 & 0.68 & .043 \\
\hline & Av vs Lar & 7.11 & 0.70 & -0.65 & 0.02 & .071 \\
\hline & Lar vs $\mathrm{Sm}$ & 7.43 & 0.57 & -0.36 & 0.31 & 1.000 \\
\hline 42 & Av vs Sm & 7.47 & 0.63 & -0.18 & 0.71 & .464 \\
\hline
\end{tabular}




\begin{tabular}{|c|c|c|c|c|c|c|}
\hline & Av vs Lar & 7.21 & 0.70 & -0.56 & 0.33 & 1.000 \\
\hline & Lar vs Sm & 7.32 & 1.11 & -0.59 & 0.30 & 1.000 \\
\hline \multirow{3}{*}{43} & Av vs Sm & 8.86 & 0.87 & -0.11 & 0.80 & .214 \\
\hline & Av vs Lar & 8.52 & 0.83 & -0.61 & 0.30 & 1.000 \\
\hline & Lar vs Sm & 8.67 & 0.91 & -0.65 & 0.27 & .935 \\
\hline \multirow[t]{3}{*}{44} & Av vs Sm & 7.63 & 0.60 & -0.12 & 0.58 & .341 \\
\hline & Av vs Lar & 7.40 & 0.71 & -0.35 & 0.35 & 1.000 \\
\hline & Lar vs Sm & 7.39 & 0.68 & -0.58 & 0.12 & .326 \\
\hline \multirow[t]{3}{*}{45} & Av vs Sm & 6.43 & 0.52 & -0.23 & 0.34 & 1.000 \\
\hline & Av vs Lar & 6.38 & 0.63 & -0.31 & 0.26 & 1.000 \\
\hline & Lar vs Sm & 6.40 & 0.47 & -0.31 & 0.26 & 1.000 \\
\hline \multirow[t]{3}{*}{46} & Av vs Sm & 6.12 & 0.45 & -0.31 & 0.22 & 1.000 \\
\hline & Av vs Lar & 6.17 & 0.52 & -0.28 & 0.25 & 1.000 \\
\hline & Lar vs Sm & 6.18 & 0.55 & -0.21 & 0.33 & 1.000 \\
\hline \multirow[t]{3}{*}{47} & Av vs Sm & 5.81 & 0.55 & -2.67 & 2.67 & 1.000 \\
\hline & Av vs Lar & 5.81 & 0.65 & -3.95 & 1.37 & .724 \\
\hline & Lar vs Sm & 7.10 & 0.72 & -1.38 & 3.96 & .732 \\
\hline
\end{tabular}

Note: *FDI, notation; LAIPG, lower arch interpremolar widths groups; SM, small arch length; AV, average arch length; LA, large arch

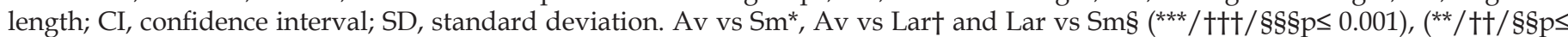
$0.01)$ and $(* / \dagger / \S p \leq 0.05)$.

Table 12: Crown height in relation to lower arch intermolar widths groups.

\begin{tabular}{|c|c|c|c|c|c|c|}
\hline \multirow[t]{2}{*}{ Variables* } & \multirow[t]{2}{*}{ LAIMW } & \multirow[t]{2}{*}{ Mean } & \multirow[t]{2}{*}{ SD } & \multicolumn{2}{|c|}{$95 \% \mathrm{CI}$} & \multirow[t]{2}{*}{$\mathbf{P}$} \\
\hline & & & & Lower & Upper & \\
\hline \multirow[t]{3}{*}{31} & Av vs Sm & 7.43 & 0.76 & -0.09 & 0.59 & .242 \\
\hline & Av vs Lar & 7.18 & 0.56 & -0.50 & 0.21 & .957 \\
\hline & Lar vs Sm & 7.32 & 0.63 & -0.45 & 0.24 & 1.000 \\
\hline \multirow[t]{3}{*}{32} & Av vs Sm & 7.46 & 0.74 & -0.23 & 0.66 & .747 \\
\hline & Av vs Lar & 7.25 & 0.58 & -0.46 & 0.47 & 1.000 \\
\hline & Lar vs Sm & 7.24 & 1.17 & -0.67 & 0.23 & .709 \\
\hline \multirow[t]{3}{*}{33} & Av vs Sm & 8.73 & 0.91 & -0.28 & 0.61 & 1.000 \\
\hline & Av vs Lar & 8.56 & 0.78 & -0.44 & 0.49 & 1.000 \\
\hline & Lar vs Sm & 8.54 & 0.88 & -0.65 & 0.25 & .876 \\
\hline \multirow[t]{3}{*}{34} & Av vs Sm & 7.40 & 0.64 & -0.19 & 0.50 & .814 \\
\hline & Av vs Lar & 7.24 & 0.70 & -0.45 & 0.26 & 1.000 \\
\hline & Lar vs Sm & 7.34 & 0.65 & -0.41 & 0.28 & 1.000 \\
\hline \multirow[t]{3}{*}{35} & Av vs Sm & 6.39 & 0.59 & -0.14 & 0.42 & .646 \\
\hline & Av vs Lar & 6.25 & 0.49 & -0.39 & 0.19 & 1.000 \\
\hline & Lar vs Sm & 6.35 & 0.51 & -0.32 & 0.24 & 1.000 \\
\hline \multirow[t]{3}{*}{36} & Av vs Sm & 6.10 & 0.44 & -0.27 & 0.24 & 1.000 \\
\hline & Av vs Lar & 6.11 & 0.50 & -0.29 & 0.24 & 1.000 \\
\hline & Lar vs Sm & 6.14 & 0.54 & -0.22 & 0.29 & 1.000 \\
\hline \multirow[t]{3}{*}{37} & Av vs Sm & 5.86 & 0.52 & -0.21 & 0.46 & 1.000 \\
\hline & Av vs Lar & 5.73 & 0.72 & -0.22 & 0.48 & 1.000 \\
\hline & Lar vs Sm & 5.60 & 0.70 & -0.59 & 0.08 & .202 \\
\hline \multirow[t]{3}{*}{14} & Av vs Sm & 7.44 & 0.74 & -0.08 & 0.60 & .194 \\
\hline & Av vs Lar & 7.19 & 0.56 & -0.51 & 0.19 & .840 \\
\hline & Lar vs Sm & 7.34 & 0.62 & -0.44 & 0.24 & 1.000 \\
\hline 42 & Av vs Sm & 7.46 & 0.73 & -0.25 & 0.62 & .912 \\
\hline
\end{tabular}




\begin{tabular}{|c|c|c|c|c|c|c|}
\hline & Av vs Lar & 7.28 & 0.58 & -0.41 & 0.50 & 1.000 \\
\hline & Lar vs Sm & 7.23 & 1.15 & -0.67 & 0.21 & .636 \\
\hline \multirow[t]{3}{*}{43} & Av vs Sm & 8.76 & 0.98 & -0.33 & 0.58 & 1.000 \\
\hline & Av vs Lar & 8.64 & 0.75 & -0.48 & 0.47 & 1.000 \\
\hline & Lar vs Sm & 8.64 & 0.88 & -0.58 & 0.34 & 1.000 \\
\hline \multirow[t]{3}{*}{44} & Av vs Sm & 7.50 & 0.65 & -0.21 & 0.48 & 1.000 \\
\hline & Av vs Lar & 7.37 & 0.66 & -0.52 & 0.20 & .841 \\
\hline & Lar vs Sm & 7.53 & 0.71 & -0.32 & 0.38 & 1.000 \\
\hline \multirow[t]{3}{*}{45} & Av vs Sm & 6.50 & 0.57 & -0.08 & 0.48 & .238 \\
\hline & Av vs Lar & 6.29 & 0.54 & -0.41 & 0.17 & .957 \\
\hline & Lar vs Sm & 6.41 & 0.49 & -0.36 & 0.20 & 1.000 \\
\hline \multirow[t]{3}{*}{46} & Av vs Sm & 6.16 & 0.44 & -0.25 & 0.28 & 1.000 \\
\hline & Av vs Lar & 6.14 & 0.56 & -0.30 & 0.24 & 1.000 \\
\hline & Lar vs Sm & 6.17 & 0.53 & -0.25 & 0.28 & 1.000 \\
\hline \multirow[t]{3}{*}{47} & Av vs Sm & 5.88 & 0.52 & -2.60 & 2.67 & 1.000 \\
\hline & Av vs Lar & 5.84 & 0.69 & -3.99 & 1.49 & .815 \\
\hline & Lar vs Sm & 7.09 & 0.05 & -1.44 & 3.87 & .806 \\
\hline
\end{tabular}

Note: *FDI, notation; LAIMW, lower arch intermolar widths groups; SM, small arch length; AV, average arch length; LA, large arch

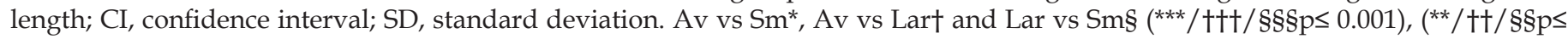
$0.01)$ and $(* / \dagger / \S p \leq 0.05)$.

\section{Discussion}

Current research investigated the crown height in relation with arch perimeter, length and width groups (intercanine, interpremolar and intermolar) for the first time [12]. investigates only mesiodistal size of tooth and size of tooth ratio in relation to these groups via CBCT acquisitions. In orthodontic diagnosis as well as treatment planning, the evaluation of tooth size and tooth size discrepancy is an indispensable step and this investigation is generally determined by conventional plaster study model analysis [10]. The crown height is of extreme value in the orthodontic bonding, the wrong bracket position can have adverse consequences on the ideal treatment goals. Current study reveals the crown height database which can be used as norms for the placement of preadjusted fixed orthodontic brackets. The responsibility of the orthodontist is to visualize the macro, mini and micro-esthetics to design a pleasant smile. The crown height has great part in facial charm.

The crown height has importance in relation to placement of orthodontic brackets bonding, also has effect on the facial height of the patient. Current study revealed the crown height of male is significantly greater than female (Tables 1-2) which are in accordance with other investigators. Our research investigates the class I ideal occlusion with ideal overjet, overbite and proper interdigitation to arch perimeter, length and width groups (intercanine, interpremolar and intermolar). The research results showed no significant difference to these groups of ideal occlusions (Tables 3-12). Other study investigated the ideal occlusion in relation to tooth size ratios and found significant difference to these groups [12] but the Crown height has no significant difference to various arch groups. Crown height is a valuable landmark for the orthodontists to manage open or deep bite cases. Thus, the current study provides, the norms, database for the crown height of Pakistani population.
Therefore, orthodontics can use crown height as a reference guide for the purpose of orthodontic intrusion and extrusion. For the Pakistani population the tooth was investigated in mesiodistal, buccolingual and diagonal dimension. Crown height of maxilla and mandible revealed for first time sexual disparities in Pakistani population. These norms shall be supportive in orthodontics clinical treatment planning, prosthodontics, periodontology and restorative dentistry. Furthermore, the norms will be of great value in forensic dentistry, and dental anthropology. As the human teeth and arch size have lots of variations in size in relation to culture, race and sex. Therefore, such investigations need to be carried out on other population.

\section{Conclusion}

a) No significant differences were observed for crown height relating to-

b) Maxillary and mandibular arch perimeter groups

c) Maxillary plus mandibular arch length groups

d) Maxillary plus mandibular arch width groups

e) Inter canine width groups

f) Inter first premolar width groups

g) Inter first molar width groups

h) Significant sexual dimorphisms were observed in the crown height of males and females.

\section{References}

1. Alam MK, Iida J (2013) Overjet, overbite and dental midline shift as predictors of tooth size discrepancy in a Bangladeshi population and a graphical overview of global tooth size ratios. Acta Odontol Scand 71(6): 1520-1531. 
2. Alam MK, Shahid F, Purmal K, Ahmad B, Khamis MF (2014) Bolton tooth size ratio and its relationship with arch widths, arch length and arch perimeter: A cone beam computed tomography (CBCT) study. Acta Odontol Scand 72(8): 1047-1053.

3. Bergman RT, John W, Ali BF, Neal CM (2013) Longitudinal study of cephalometric soft tissue profile traits between the ages of 6 and 18 years. Angle Orthod 84(1): 48-55.

4. Crosby, Douglas R, Charles GA (1989) The occurrence of tooth size discrepancies among different malocclusion groups. Am J Orthod Dentofacial Orthop 95(6): 457-461.

5. Djeu G, Catherine H, Samer Z (2002) Correlation between mandibular central incisor proclination and gingival recession during fixed appliance therapy. Angle Orthod 72(3): 238-245.

6. Dupont WD, Walton DP (1998) Power and sample size calculations for studies involving linear regression. Contr clinic tri 19(6): 589-601.

7. Endo T, Ryota A, Hiroo K, Kenji Oka, Shohachi Shimooka (2008) Tooth size discrepancies among different malocclusions in a Japanese orthodontic population. Angle Orthod 78(6): 994-999.

8. Hasanreisoglu U, Semih B, Kerem A (2005) An analysis of maxillary anterior teeth: facial and dental proportions. J Prosthet Dent no 94(6): 530-538.

9. Houston WJB (1983) The analysis of errors in orthodontic measurements. Am J Orthod no 83(5): 382-390.

10. Kansal A, Nandan K, Kanhoba MK (2012) Analysis of Bolton's ratio among different malocclusion groups: A hospital based study. Indian J Dent no 3(3): 139-144.

11. Lavelle CLB (1972) Maxillary and mandibular tooth size in different racial groups and in different occlusal categories. Am J Orthod no 61(1): 29-37.

12. Malik SN, Alam MK, Khamis MF, Pavan Gothe (2014) Racial dimorphism in Indians and Malaysians based on tooth size. Int Med J no 21(1): 31-33.

13. Mihailidis S, Graham S, Khamis MF, Grant T (2013) Prevalence and patterning of maxillary premolar accessory ridges (MxPARs) in several human populations. Am J Phys Anthropol no 152(1): 19-30.

14. O Mahony G, Declan TM, Mark KB, McIntyre GT, Cronin MS (2011) Tooth size discrepancies in Irish orthodontic patients among different malocclusion groups. Angle Orthod no 81(1): 130-133.

\section{ISSN: 2574-1241}

DOI: $10.26717 /$ BJSTR.2018.12.002208

Mohammad Khursheed Alam. Biomed J Sci \& Tech Res

This work is licensed under Creative

Commons Attribution 4.0 License

Submission Link: https://biomedres.us/submit-manuscript.php
15. Owens, Edward G, Charles JG, Okamura M, Jo KH, et al. (2002) A multicenter interracial study of facial appearance. Part 2: A comparison of intraoral parameters. Int J Prosthodont no 15(3): 283-288.

16. Prabhu S, Ashith BA (2009) Odontometric sex assessment in Indians. Forensic Sci Int no 192(1-3): 129 e1-129 e5.

17. Purohit Z, Santosh KG, Narayan K (2012) Is clinical crown height a marker of lower anterior facial height. J Dent Oral Biosci no, p. 3.

18. Ribeiro DC, Brook AH, Hughes TE, Sampson WJ, Townsend GC (2013) Intrauterine hormone effects on tooth dimensions. J Dent Res no 92(5): 425-431.

19. Shahid F, Alam MK, Khamis MF, Yuma Honda (2015) Geomorphometric of tooth size and arch dimension analysis by conventional digital caliper and digital stereomicroscope to establish standard norms for the Pakistani population. J Hard Tiss Biol no 24(2): 155-168.

20. Shahid F, Alam MK, Khamis MF (2014) Validity and reliability of digital model measurements: A digital stereomicroscopic study. J Hard Tiss Biol no 23(4): 439-444.

21. Sterrett JD, Trudy O, Fonda R, Weston F, Ben K, et al. (1999) Width/length ratios of normal clinical crowns of the maxillary anterior dentition in man. J clin Periodont no 26(3): 153-157.

22. Ta TA, John YKL, Urban H (2001) Tooth-size discrepancies among different occlusion groups of southern Chinese children. Am J Orthod Dentofacial Orthop no 120(5): 556-558.

23. Townsend G, Michelle B, Toby H, Alan B (2012) Genetic, environmental and epigenetic influences on variation in human tooth number, size and shape. Odontol no 100(1): 1-9.

24. Vandenbroucke JP, Erik VE, Douglas GA, Peter CG, et al. (2007) Strengthening the Reporting of Observational Studies in Epidemiology (STROBE): explanation and elaboration. PLoS medicine no 4(10): e297.

25. Volchansky A, Peter CJ (2001) Clinical crown height (length) a review of published measurements. J Clin Periodont no 28(12): 1085-1090.

26. Williams AC, Stephens CD (1992) A modification to the incisor classification of malocclusion. Brit J Orthod no 19(2): 127-130.

27. Yonezu T, John JW, Samir EB, Kari LS (2001) Comparison of Tooth Size and Dental Arch Widths in Contemporary Japanese and American Preschool Children. W J Orthod no 2(4): 356-360.

Cite this article: Fazal Shahid, Mohammad Khursheed A, Mohd Fadhli K, Saqib A. Crown Height and its Relation to Arch Width, Arch Length 